\title{
Upgrading the capacity and skills of service providers of the West Bank/Gaza Pilot Health Project
}

\author{
Nancy Ali \\ Laila Nawar \\ Dale Huntington \\ Tawhida Khalil
}

Follow this and additional works at: https://knowledgecommons.popcouncil.org/departments_sbsr-rh

Part of the Demography, Population, and Ecology Commons, Health Services Research Commons, International Public Health Commons, and the Maternal and Child Health Commons How does access to this work benefit you? Let us know!

\section{Recommended Citation}

Ali, Nancy, Laila Nawar, Dale Huntington, and Tawhida Khalil. 2002. "Upgrading the capacity and skills of service providers of the West Bank/Gaza Pilot Health Project," FRONTIERS Final Report. Washington, DC: Population Council. 


\section{Upgrading the Capacity and Skills of Service Providers of the West Bank/Gaza Pilot Health Project \\ Nancy Ali, MPH, Laila Nawar, Ph.D., Dale Huntington, Sc.D., Tawhida Khalil, Ph.D.}

August 2002

This study was funded by the U.S. AGENCY FOR INTERNATIONAL DEVELOPMENT (USAID) under the terms of Cooperative Agreement number HRN-A-00-98-00012-00 and subagreement number AI00.40A. The opinions expressed herein are those of the authors and do not necessarily reflect the view of USAID. 


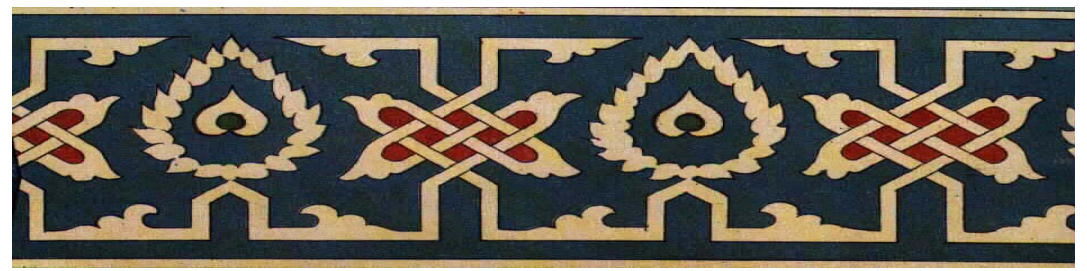

\section{SUMMARY}

The insufficient care that mothers and newborns receive during the prenatal period is one of the most pressing health care problems in the West Bank and Gaza. Although a large proportion of mothers receive some antenatal care, only about 17 percent return for postpartum check-ups and few adopt family planning for spacing births during the postpartum period (PRC, 1996). Fertility rates in the West Bank and Gaza are higher than other countries with similar development status in the region.

To address the peri-natal needs of Palestinian women, the United States Agency for International Development Mission for the West Bank and Gaza, in collaboration with the Palestinian Ministry of Health and seven partner organizations, designed and funded a 28-month pilot health project that is expected to have a significant health impact on Palestinian women and their children. The goal of this Pilot Health Project (PHP) is to improve the health status of Palestinian women and their children by upgrading antenatal and postpartum services in three areas in the West Bank and Gaza; specifically in Jenin, Hebron and Gaza. One of the key components of the PHP was the design and implementation of a comprehensive training program aimed at upgrading the capacity and skills of PHP health care providers at 27 clinic sites in the West Bank and Gaza.

Center for Development in Primary Health Care (CDPHC), in collaboration with the Population Council's FRONTIERS Program, developed a 19-month training program to strengthen and improve existing maternal and children's health and family planning services and to promote the establishment of the standardized basic package of services at PHP clinic sites. There were four key start-up activities, including: 1) an assessment of standards of care provided at PHP clinics; 2) a review of formative research; 3 ) the development of clinical training manuals and curricula; and 4) the development of behavior change communication (BCC) materials. In June 2000, a comprehensive training strategy and program was developed. The program's strategy was based primarily on an assessment of the existing standards of care provided at PHP clinics, carried out in August 1999. The findings of this assessment revealed that the knowledge 
and skills of all cadres of providers (physicians, nurses and community health workers) at PHP clinic sites needed upgrading.

The program's components included a Training of Trainers workshop, in-service training, development of a monitoring and supervision system, and follow-up refresher training. Built-in evaluation mechanisms, including an on-site and off-site monitoring and supervision system (whole-site training supervision approach), were developed and established by the CDPHC.

In June and July 2001, CDPHC conducted a post-training evaluation to measure the impact of their training program. The results from this evaluation assisted in determining the needs for refresher training and identifying gaps and weaknesses that continued to exist in the knowledge and skills of health providers previously trained under the PHP. This evaluation included in-depth interviews with staff, an observation checklist to evaluate the skills and performance of health care providers, and focus groups discussions with clients. Although health providers reported that the training improved their knowledge and interpersonal skills, about half of the participants (mostly community health workers) reported that they needed more training in additional topics (e.g., maternal and child psychology).

Refresher training workshops were organized and conducted by CDPHC for all cadres of health providers. The curricula for the refresher training courses were designed based on the feedback/data collected from the monitoring and supervision field visits.

In order to promote quality services provided to clients, however, a number of workrelated barriers still need to be overcome. These barriers include lack of transportation, difficulties in conducting home visits because the coverage areas are too large, and inadequate physical conditions at the clinics (e.g. shortage of equipment, inadequate space and staff, lack of electricity, water supply and/or telephone services).

In conclusion, the training provided by CDPHC for the PHP has positively influenced the level of knowledge and skills of health providers as well as the quality of services provided at the PHP clinics. Nevertheless, more training is needed to strengthen existing health care services at 
the primary level and reduce the gaps and weaknesses identified by the post-training evaluation. Furthermore, clients and medical staff need a suitable physical environment for the provision of quality services, which requires a better level of preparedness of health facilities. Through the PHP, a number of lessons were learned and recommendations were raised. The most important lessons learned and conclusions from the PHP include the following:

\section{Lessons learned}

을 The development of a physical facility checklist would help optimize and standardize the quality of service.

Q Doctors' sense of safety is a priority in order for them to move between health facilities.

응 A highly participatory and inclusive management strategy is essential for training programs that involve multiple service delivery organizations in order to meet everyone's expectations and particular needs.

0 Ongoing training is essential in order to sustain and improve the knowledge and skills of health care providers.

[ Implementing agencies need to be flexible given the unstable environment and travel restrictions. Contingency plans for program and/or budget changes need to be developed to work around barriers and accomplish activities.

\section{Recommendations}

D. Additional Behavior Change Communication (BCC) materials, such as brochures, posters, and audio-visual aids, need to be distributed to PHP clinics to complement the existing counseling sessions by clinic staff and to improve the quality of health education and counseling services.

을 Supportive supervision mechanisms (on-site supervisors, monitoring and supervision visits) need to be established and maintained. Supportive supervision visits should focus on constructive feedback, coaching, encouragement, understanding of constraints, and help with problem-solving.

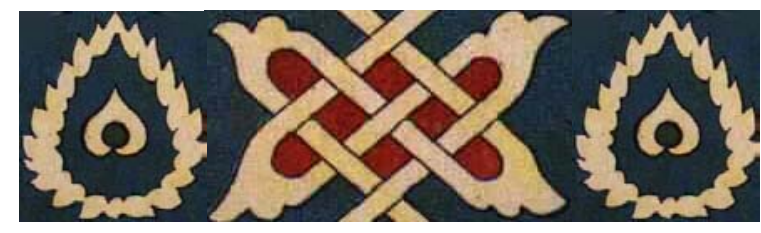




\section{CONTENTS}

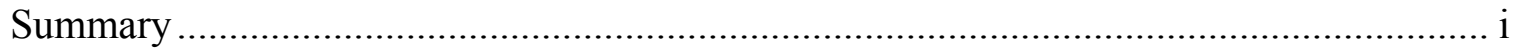

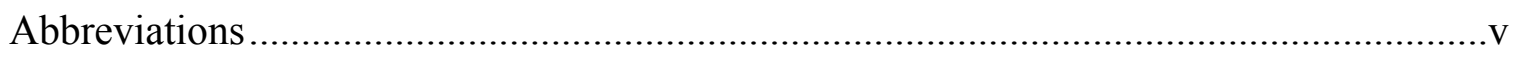

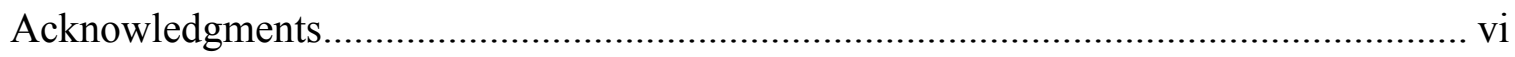

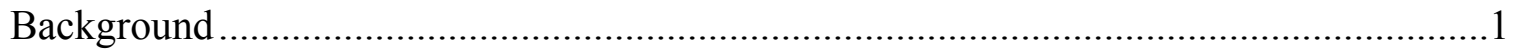

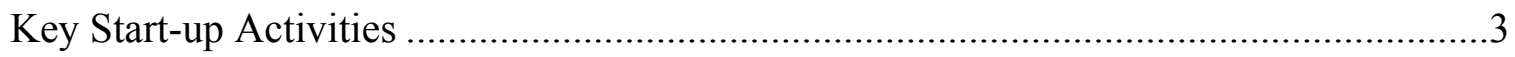

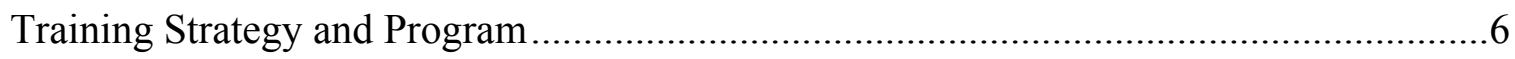

Rapid Assessment of Providers' Expectations ..................................................... 8

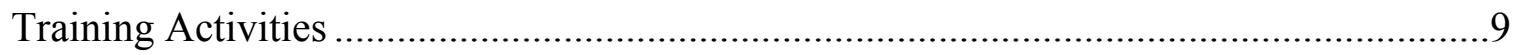

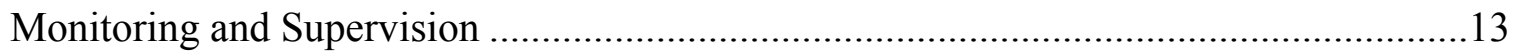

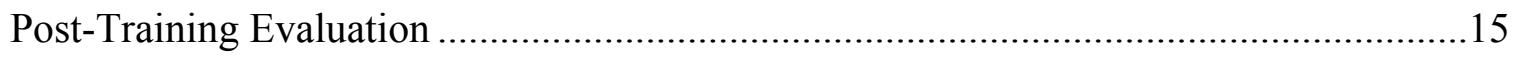

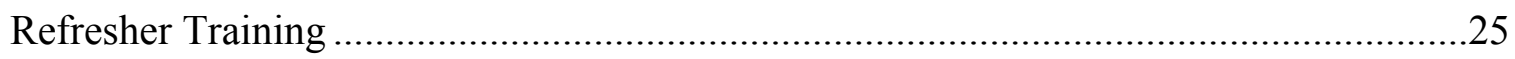

Conclusions and Recommendations ......................................................................2 


\begin{tabular}{ll} 
ABBREVIATIONS \\
BCC & Behavior Change Communication \\
CDPHC & Center for Development in Primary Health Care \\
IMR & Infant Mortality Rate \\
MMR & Maternal Mortality Rate \\
MOH & Ministry of Health \\
NGO & Non-governmental Organization \\
PCBS & Palestinian Central Bureau of Statistics \\
PHP & Pilot Health Project \\
PRC & Planning and Research Center \\
TOT & Training of Trainers \\
TRC & Technical Review Committee \\
UNWRA & United Nations Relief and Works Agency for Palestine Refugees in the Near East \\
USAID & United States Agency for International Development \\
\hline
\end{tabular}




\section{ACKNOWLEDGMENTS}

Center for Development in Primary Health Care/Al Quds University (CDPHC) represented by its director Prof. Mohammed Shaheen, would like to thank all those who participated in this program and made it possible for CDPHC to compile all the needed information, namely; doctors, nurses, community health workers, female clients of the Union of Medical Relief Committees, the Patient Friends Society and the Union of Health Work Committees.

CDPHC would also like to thank the two on-site supervisors, Ms. Tamam Shalabi and Ms. Muna Zaqout, as well as Dr. Yousef Sweetih, who participated in conducting the follow-up clinic and home visits. They interviewed and observed female clients and providers and assessed whether or not providers complied with the training protocols and guidelines utilized throughout the life of the project.

CDPHC would like to thank Dr. Rand Jarallah for her contribution in developing the indepth interviews and observation checklist that were used during the assessment of the health care providers.

CDPHC would like to thank Dr. Khaleel Suleiman for hosting some of the meetings and interviews at his private clinic in Jenin.

CDPHC would like to thank the Population Council's Frontiers in Reproductive Health Program staff for their technical support throughout all project phases. Finally, CDPHC would like to thank USAID for funding the training component of the Pilot Health Project. 


\section{BACKGROUND}

The insufficient care that mothers and newborns receive during the prenatal period is one of the most pressing health care problems in the West Bank and Gaza today. Although a large proportion of mothers receive some antenatal care, only about 17 percent return for postpartum check-ups and few adopt family planning for spacing births during the postpartum period (PRC, 1996). Fertility rates in the West Bank and Gaza are higher than other countries in the region of similar development status. Married women in Gaza have an average of 6.8 children, while those in the West Bank have 5.5 (PCBS, 1997). Cultural norms favor early marriage, sons, and a large family. Since 46.9 percent of the West Bank and Gaza population is less than 15 years old, there will be substantial population momentum even if birth rates fall during the coming years (PCBS, 1997).

Although current levels of modern contraceptive use (estimated at 38.8\% in West Bank and $32.7 \%$ in Gaza) are fairly impressive, they tend to mask wide variations of use. For example, in parts of East Jerusalem, the contraceptive prevalence rate is 50 percent whereas it is near zero in some isolated areas of the West Bank. Contraceptive use is very low among younger, lowparity women and is generally only acceptable after parity 4 for most families (PCBS, 2000).

Current data from the West Bank and Gaza also indicate that the estimated infant mortality rate (IMR) ranges from 25.5 to 31.2 per 1,000 live births with regional differences (UNFPA, 2000). Neonatal mortality as a proportion of the IMR has been increasing (a condition also noticed in countries such as Egypt and Morocco) with pre-maturity of newborns being the preeminent cause. The maternal mortality ratio (MMR) is estimated to range between 70 and 80

per 100,000 live births. These IMR and MMR rates are comparatively lower than prevailing rates in most other countries in the region. However, the Palestinian Ministry of Health (MOH) estimates that pregnancy and maternity-related complications are the third leading cause of death among Palestinian women of reproductive age (PMOH, 1997). 


\section{Pilot Health Project (PHP)}

To address the above needs and concerns, the United States Agency for International Development (USAID) Mission for the West Bank and Gaza, in collaboration with the $\mathrm{MOH}$ and seven partners organizations, designed and funded a 28-month pilot health project that is expected to have a significant health impact on Palestinian

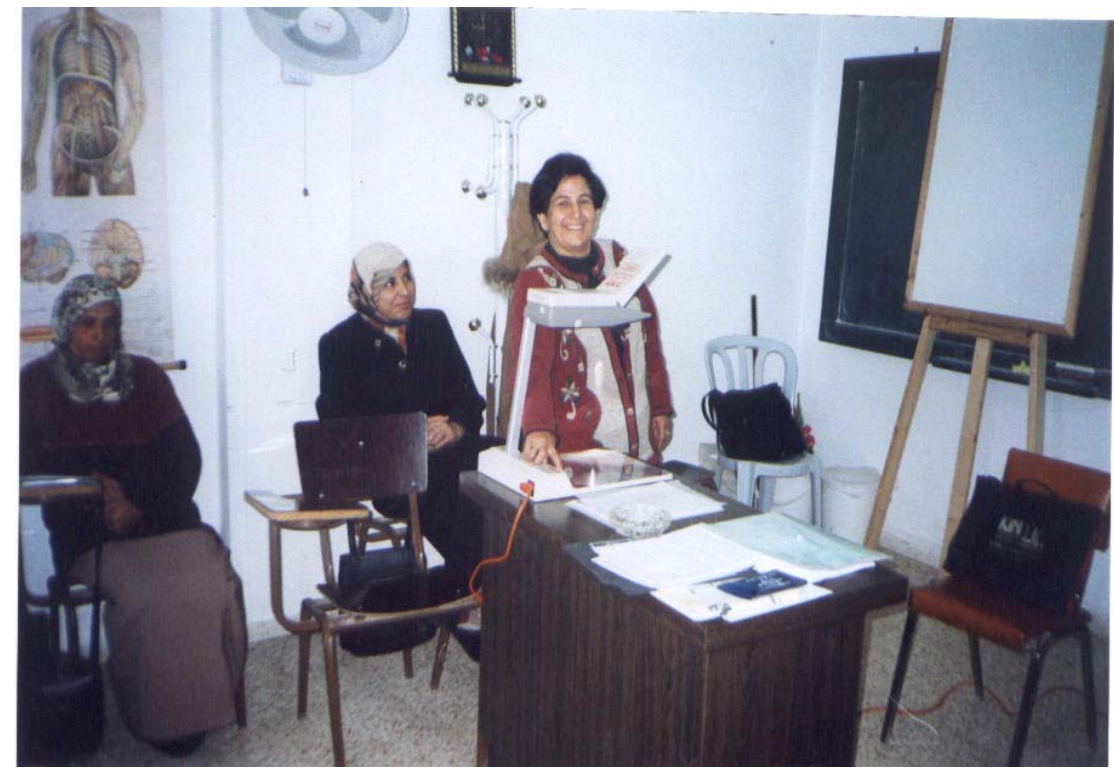
women and their children. The goal of the PHP is to improve the health status of Palestinian women and their children by upgrading antenatal and postpartum services in three areas in the West Bank and Gaza; specifically, Jenin, Gaza and Hebron. The project focuses on the provision of a basic package of services offering quality antenatal and postpartum care (as shown in the Basic Package of Services text box) as well as three research interventions aimed at improving the health-giving practices of providers and health-seeking behavior of families.

The estimated number of women of fertile age in the three project areas are: 35,000 in Jenin; 20,000 in Gaza; and 20,500 in Hebron. It is expected that about 18,000 women and children will receive upgraded services during the 28 months of the PHP.

- Improved antenatal care through the NGO clinic.

- A home visit by the NGO's community health worker two or three days after discharge from the hospital or after delivery at home (for mothers who give consent). The community health worker will assess the health condition of both the mother and the newborn and provide health education about breastfeeding and nutrition, care of the newborn, and immunization.

- Counseling on birth spacing and the need for postpartum contraception.

- Encouragement for mothers to return to the clinic with their newborns on the $40^{\text {th }}$ day after delivery for a postpartum check-up.

One of the key components of the PHP was the design and implementation of a comprehensive training program aimed at increasing the capacity and upgrading the skills of PHP health care personnel. The Center for Development in Primary Health Care (CDPHC) in 
collaboration with the Population Council's FRONTIERS Program developed a 19-month training program to strengthen and improve existing maternal and children's health/family planning (MCH/FP) services and to promote the establishment of the standardized basic package of services at PHP clinic sites.

The training program's strategy was based upon an assessment of the existing standards of care provided at PHP clinics which was carried out in August 1999. The findings of this assessment revealed that the knowledge and skills of all cadres of providers (i.e. physicians, nurses and community health workers) at PHP clinic sites needed upgrading. Improving training and supervision of PHP clinic staff to provide safe, high-quality services was seen as key to increasing client utilization of antenatal, postpartum and family planning services as well as to having a lasting impact on the PHP objectives and indicators.

\section{Setting}

The training program targeted clinic staff and clients working in and attending the following 27 PHP clinic sites: six Union of Medical Relief Committees (UPMRC) clinics in Jenin and Hebron; five Union of Health Work Committees (UHWC) clinics in Gaza; and 16 Patient Friends Society (PFS) clinics in Jenin. The program also included MOH personnel, where feasible.

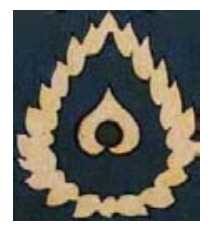

\section{KEY START-UP ACTIVITIES}

To ensure that training activities for the PHP commenced as quickly as possible, CDPHC coordinated several key start-up activities for the training program in late 1999. These activities were essential building blocks in the development of the 19-month training program and provided the PHP clinics with vital training materials and behavior change communication (BCC) messages and materials.

\section{Assessment of Standards of Care Provided at PHP Clinics}

In order to determine PHP clinics' readiness to provide improved antenatal and postpartum care services, and to identify the training needs of staff, an assessment of the existing standards from a sample of PHP clinics was conducted in August 1999. The "Assessment of Standards of Health Care Services" was conducted by two Egyptian consultants (Younis and 
Hassanein, 1999). The World Health Organization (WHO) standards of care for primary health care services were used as the framework for the review. A total of 15 PHP clinics from the three catchment areas of the PHP were selected for site visits, including: nine from Jenin; two from Hebron; and four from Gaza. The assessment provided information on ways to improve service delivery at PHP clinic sites as well as information on the level of knowledge and skills of providers. Information was collected through interviews with managers, observations of nurses' and physicians' practical skills, self-administered questionnaires, client exit interviews, and observation of home visits (outreach activities).

The results of this assessment indicated a need to update the technical knowledge and skills of all cadres of providers. The assessment indicated a gap between existing and targeted levels of quality of $\mathrm{MCH} / \mathrm{FP}$ health care. Areas of particular need included: essential antenatal and postpartum care; counseling; interpersonal communication; infection control; quality assurance and management procedures. The team also recommended revising the service delivery protocols for postpartum outreach services.

\section{Review of Formative Research}

CDPHC conducted a review of existing formative research on reproductive health and $\mathrm{MCH} / \mathrm{FP}$ issues and concerns in Palestine in late 1999. Findings and insights on the knowledge, attitudes and practices of Palestinians (with respect to maternal and child health care, pregnancy and childbirth practices, postpartum care and birth spacing) were also used to formulate the clinical training manual and curricula for the PHP training program. The review provided valuable information and guidelines for developing prototype BCC messages and materials for the PHP.

\section{Development of Clinical Training Manual and Curricula}

A committee of training experts commissioned by CDPHC used the findings from the assessment of the standards of care provided at PHP clinics as well as formative research to produce a comprehensive training manual for the PHP training program. Up-to-date scientific information on reproductive health as well as current research reports, publications, training guidelines and materials from other collaborating agencies (e.g. EngenderHealth, Pathfinder, INTRAH, FHI) and the "Contraceptive Technology Update" series were used to produce a draft 
high-quality PHP training manual. These materials were also used to develop tailored training packages for each category of staff to be trained.

CDPHC worked closely with the Technical Review Committee (TRC) members and key advisors to incorporate the information from these publications into the draft training manual and materials. The TRC included members from each PHP partner organization that provided services, representatives from the relevant directorates within the $\mathrm{MOH}$, the Population Council, and Al Quds University. Key topics covered in the manual's modules were:
영 Protocols for antenatal, postpartum and neonatal care
잉 Family planning and well-baby care
는 Nutrition and breastfeeding policies and practices
을 Breast and cervical cancer prevention and referral procedures
응 Male involvement
을 Reproductive health indicators
웅 Communication and counseling
Infection control
Q Quality management and clinical supervision
- Training of trainers (TOT) methods and tools

The manual was finalized after being pre-tested during the TOT workshop and was used for all in-service training programs for the PHP. One of the key features of the manual is that it can be tailored to each category of health provider and adapted for various types of training activities. Copies of the manual were distributed to all management staff of all partner service delivery NGOs for internal use and to the $\mathrm{MOH}$, key donors, NGOs and universities in both the West Bank and Gaza.

\section{Development of Behavior Change Communication (BCC) Materials}

$\mathrm{CDPHC}$ used the findings of the formative research along with samples of BCC materials produced by other USAID collaborating agencies in Jordan and Egypt (from projects similar to the PHP) to design and develop prototype BCC materials that support the PHP's objectives. A total of six prototype materials were developed: two posters (one for providers and one for clients) a sticker on infection prevention that goes on washroom mirrors; a fact sheet for clients 
on warning signs during pregnancy; and two client leaflets - one on gestational diabetes and one on postpartum care. The topics chosen for the prototypes were based on the existing needs at the clinic sites and complement other BCC materials already available. The materials were pretested through focus groups and spot interviews at health care facilities, and were reviewed and approved by all PHP partners. The materials were printed and disseminated to the 27 PHP clinics and are currently being used by service providers during counseling sessions. All categories of providers were trained in their use.

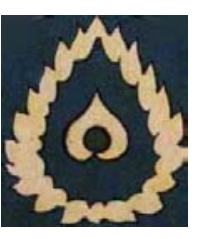

\section{TRAINING STRATEGY AND PROGRAM}

In June 2000 CDPHC with technical assistance from the Population Council produced a comprehensive training strategy and work-plan for conducting a series of in-service training activities for all PHP service providers. The overall goal of the training program was to improve the health of Palestinian women and their children. Specific objectives included:

1. Improving the quality of antenatal, postpartum, and neonatal health care and family planning services at PHP clinic sites.

2. Increasing the use of health care services by women of reproductive age and newborns through health information and home care.

3. Developing standardized case management practices for antenatal, postpartum, neonatal care and family planning for clients at PHP clinics.

These objectives can be achieved by:

을 Strengthening the delivery of a standardized package of quality antenatal and postpartum services (basic package of services described in PHP concept paper).

믈 Building the capacity of the health care personnel in order to enable the provision of safe, high-quality services.

는 Increasing client use of antenatal, postpartum and family planning services through BCC materials.

The training program employed a participatory approach and included a TOT workshop, in-service training, a monitoring and supervision system, and follow-up refresher training. 
Training activities were conducted with the oversight and guidance of senior staff from all partner agencies through the TRC. The TRC met monthly during the first year of the program to review training tools and materials and to provide input on activities for all phases of the training program.

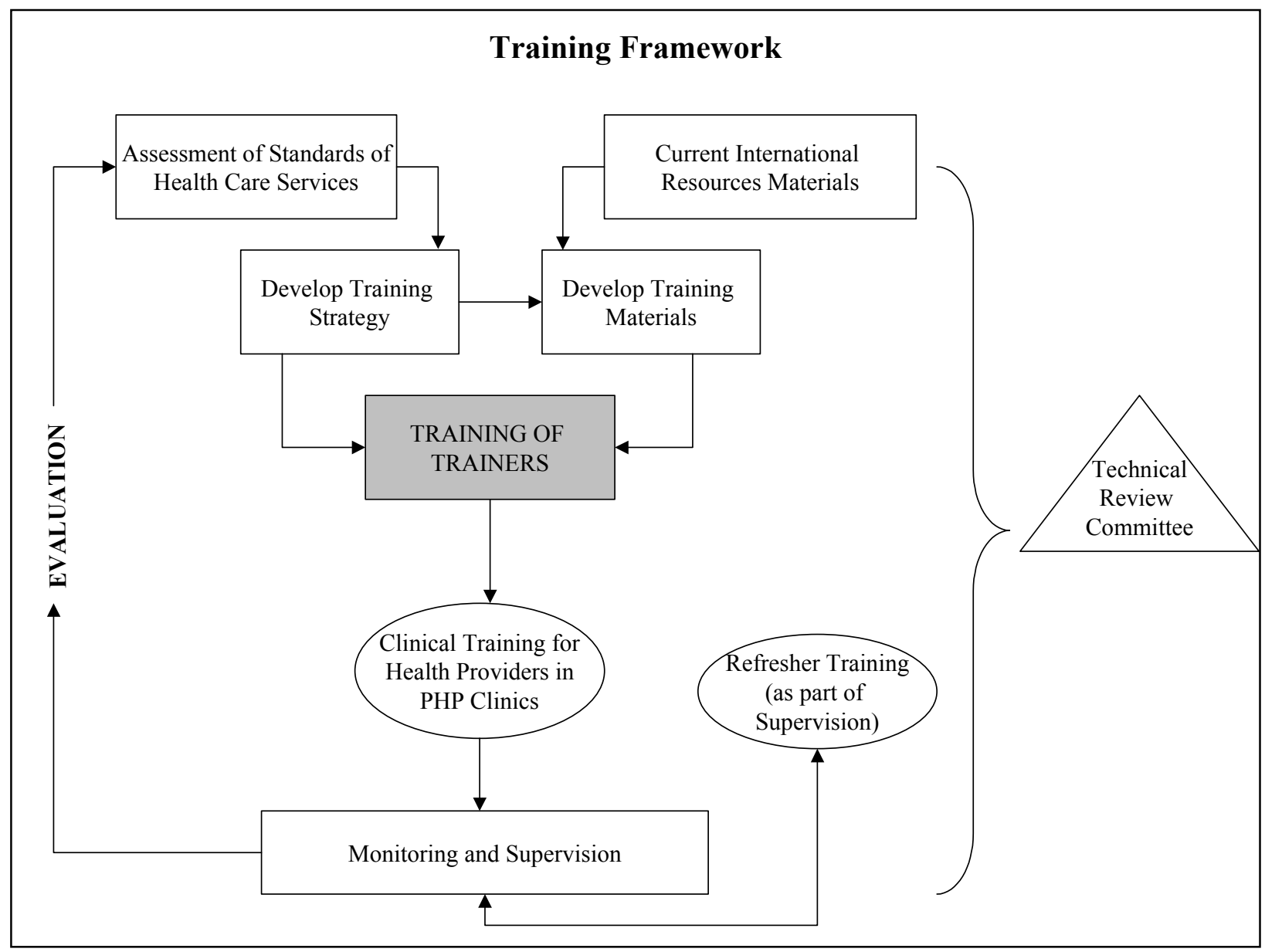

The above chart outlines the framework that was used to conduct the training in antenatal and postpartum care, family planning, counseling and quality management for the PHP. 


\section{RAPID ASSESSMENT OF PROVIDERS' EXPECTATIONS}

In keeping with the PHP's value of participation in all decision-making activities, CDPHC conducted a rapid assessment of providers' expectations for the training program. The results of this exercise were used to reach a consensus among all of the program's participants so as to increase their motivation to attend the courses. CDPHC developed a short self-administered questionnaire for providers to complete. The questionnaire was distributed to all PHP clinics and was used to solicit individual providers' recommendations for the training. CDPHC staff reviewed the completed questionnaires and conducted targeted briefings with a few providers to obtain more detailed information.

CDPHC utilized this information along with the findings and recommendations obtained from the "Assessment of Standards of Health Care Services" to help identify the training needs of each category of health provider. The assessment of standards of care offered key information on the barriers and gaps in the provision of services at PHP clinic sites and on the level of knowledge and skills of providers. The rapid assessment provided key information on providers' expectations for the training programs and complemented the review of service delivery standards.

The results of the rapid assessment provided specific information that helped shape the content of the training program. Similar to the findings from the assessment of standards of care, the rapid assessment revealed that the knowledge and skills of all cadres of providers needed upgrading. Particularly, the following areas were identified as needing improvement: essential antenatal and postpartum care; counseling; interpersonal communication; infection control; and quality assurance and management. Further, information was gathered that helped with the logistical preparations and planning for the training workshops and sessions (e.g., the appropriate length of a training day, the training language to be used, practical versus theoretical training).

A second activity of the rapid assessment involved contacting management staff of the partner organizations to enlist their support for the training program. CDPHC held meetings with senior staff from the three service delivery partner organizations, the MOH and UNWRA to 
ensure that all administrative and logistical arrangements were organized in a manner that was conducive to obtaining the active participation of staff from the service delivery organizations. The meetings also helped identify training sites, determine the sequence of training workshops (e.g., one five-day session or five one-day sessions over a longer period), and select both trainers and health providers to be trained.

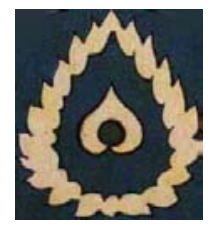

\section{TRAINING ACTIVITIES}

The clinical training offered by CDPHC focused on teaching all categories of health providers to deliver safe, high-quality antenatal, postpartum and family planning services, and selected reproductive health services to clients. CDPHC, with technical assistance from the Population Council, developed a training package that focused on essential information and clinical skills that providers could immediately use to improve the quality of their services. The main components of this training program are:

1. TOT workshop to develop a cadre of clinical "I feel many of the health care providers who did not have the chance to participate in this training trainers;

2. Training of service providers to train physicians, nurses and community health program missed a valuable opportunity to gain new skills, updated information, new techniques and methodologies." workers;

3. Development of monitoring and supervision systems; and,

4. Follow-up refresher training.

\section{TOT workshop}

The development of a cadre of clinical trainers was an essential component of the PHP training program, especially given the high turnover rates of service providers at the clinic level, estimated at 20 percent. CDPHC selected a total of 16 trainers and two alternates from

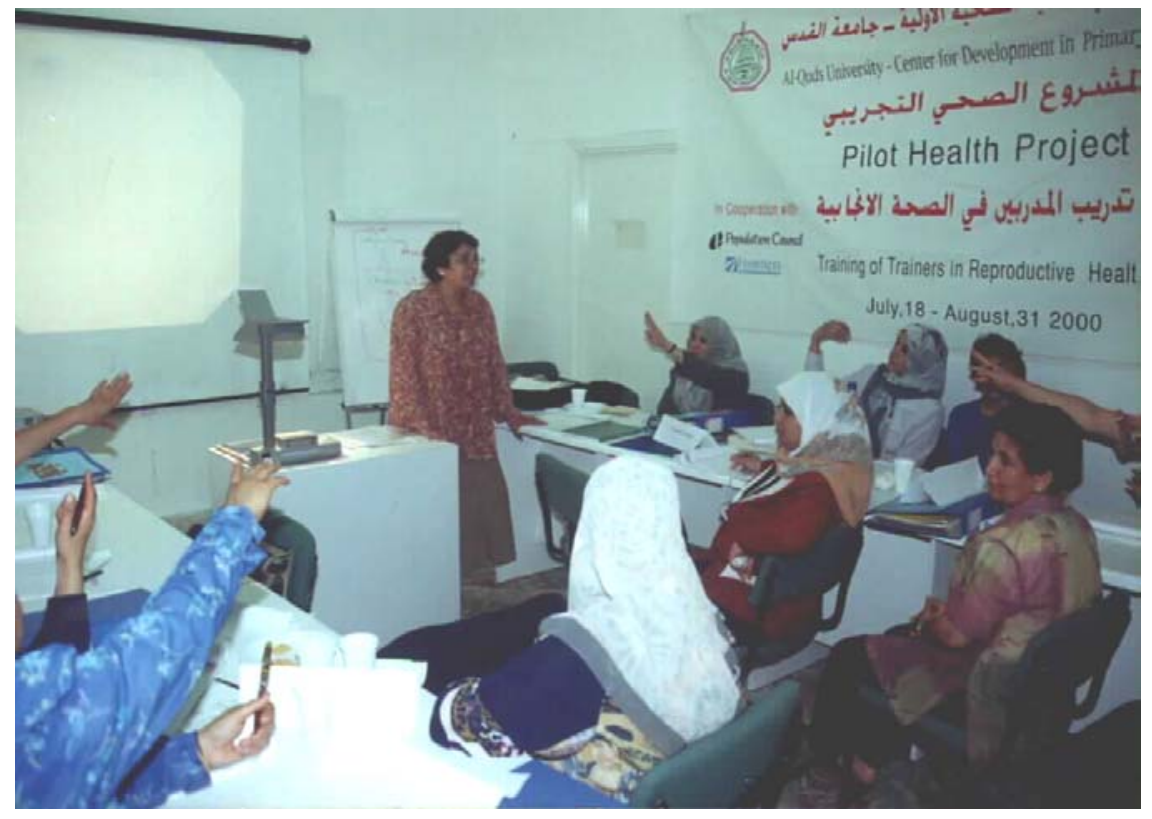


the PHP service delivery NGOs, as well as the MOH and UNRWA to participate in a TOT workshop. In order to ensure that the workshop produced a cadre of clinical trainers that formed a sustainable reproductive health training team among the three PHP service delivery agencies, the program adhered to strict selection criteria for potential trainers. Each of the trainers met the following criteria:

1. Full-time employees at one of the target service delivery NGOs (also applied to trainers from the MOH and UNWRA);

2. Experienced (proficient) in providing antenatal and postpartum care and family planning services in clinical settings;

3. Experienced in providing clinical training to others;

4. Good communication and personal skills;

5. Working knowledge of English;

6. Committed and interested in staying involved in the project and in training other staff (after completing their own training); and,

7. Committed to attending all sessions of the training.

A group of highly qualified and well-known Palestinian master trainers with expertise in various disciplines of public health conducted a TOT workshop in August 2000. A total of 13 providers were trained, including physicians, nurses, midwives and community health workers from the three partner agencies and the $\mathrm{MOH}$ over a period of 20 days. The TOT course was held at one venue for both West Bank and Gaza participants since this was more efficient and less costly than using multiple venues. The TOT course used participatory methods and focused on instilling the necessary skills to transfer expertise to others effectively, including coaching and humanistic training techniques. TOT participants' clinical skills and knowledge were also updated and standardized. By the end of the TOT, participants were able to:

1. Identify the main reproductive health indicators.

2. Apply upgraded, standardized protocols and guidelines for antenatal, postpartum, and neonatal care, infection control, and family planning.

3. Demonstrate good counseling and communication skills, especially in the areas of antenatal and postpartum care, and family planning. 
4. Provide integrated breast and cervical cancer early detection services and referral procedures at the clinical level.

5. Highlight and support the importance of male participation and involvement in reproductive health services.

6. Apply quality management and clinical supervision principles.

7. Organize and conduct training workshops for health providers.

Because most of the physicians, nurses or health workers at the clinic sites were also managers, the training of trainers program also offered instruction on management and supervision skills.

Once trained, the newly qualified trainers had to train the health providers from the PHP clinic sites. Whenever possible, and especially at the beginning of the program, the new trainers conducted these trainings with the assistance of the advanced or master trainers who taught the TOT course. This helped ensure the quality of the training provided.

\section{Training of Service Providers}

Due to the onset of the Intifada in September 2000 (and the consequent travel restrictions and closures of many areas in the West Bank and Gaza), the organization and implementation of training workshops for PHP providers was decentralized to the local level. CDPHC, with technical assistance from the Population Council, developed a contingency plan that included hiring "It is great to learn about all these new concepts and issues like Total Quality Management and MIS. This training will not only add to my skills but will improve my way of thinking on professional and personal levels."

qualified training coordinators to work with

Nurse in UHWC, Gaza available TOT graduates to conduct all training workshops at local venues in the West Bank and Gaza. Where feasible, the support of master trainers, TOT graduates, MOH representatives, and other relevant staff from the service delivery NGOs, were solicited to help monitor and supervise the training activities and to ensure the training conducted was of high quality.

Training sessions were conducted according to adult learning principles. Master and new trainers used interactive, relevant, and practical methods of training, which included behavior 
modeling, competency-based training and humanistic training techniques (e.g., anatomic models and other learning aids). The training also emphasized the use of the BCC materials developed by CDPHC. By the end of their training, health providers were able to:

을 Provide antenatal, postpartum, and family planning services according to improved and upgraded protocols and standards.

믈 Provide integrated breast and cervical cancer early detection services and referral procedures at the clinical level.

[0 Demonstrate the use of reproductive health indicators in providing services.

[0] Demonstrate good infection control standards and practices.

을 Demonstrate good counseling and communication skills, especially in the areas of antenatal and postpartum care and family planning.

을 Highlight the importance of male participation and involvement in reproductive health services.

Apply quality management and clinical supervision principles.

Community health workers received a more expanded training course than the other health care providers since they made up the majority of staff at the clinics and were responsible for conducting all outreach activities. Their training included an emphasis on the use of BCC materials developed by CDPHC. By the end of their training, community health workers were able to:

을 Provide upgraded and improved postpartum care services at the clinic and home visit levels.

D. Demonstrate good counseling and health education skills for antenatal and postpartum care (including nutrition and breastfeeding) and family planning.

[0] Teach clients how to conduct self-examinations for early detection of breast cancer.

을 Demonstrate good infection control practices at both the clinic sites and during home visits.

들 Conduct participatory rapid assessments and/or community needs assessments.

을 Demonstrate proper reporting and record keeping practices. 
A total of 31 community health workers from PFS, the MOH and UHWC were trained in the West Bank and Gaza (18 in Gaza and 13 in Jenin) during the last quarter of 2000. A second group of community health workers (19 participants from PFS and UPMRC) received training in January 2001. In total, 50 community health workers were trained under the PHP. Eighteen physicians and nurses were trained in the West Bank and Gaza in April 2001.

Each trainee's technical knowledge and skills on topics covered during the course were assessed before and after each training workshop. Trainers used observation checklists to evaluate each trainee's clinical skills and competence. The checklists focused on key steps or tasks and allowed the trainer to objectively evaluate and record the overall performance of each skill or activity. The checklist evaluation formed the basis for follow-up feedback and also assisted in identifying trainees that required supportive supervision and perhaps refresher training courses.

Anecdotal feedback on the training participants coupled with the training program's preand post-test results indicated that the trainees acquired a large amount of new information and new skills. Trainees liked the participatory approach utilized by CDPHC and its trainers, which was new to many of them. Most of the trainees reported that the training workshops boosted their confidence and changed many of

"My work performance improved and I tried to create new things in my work from the information and skills I gained from the training. In addition training refreshed my information and promoted self-trust and people respect my work."

Community health worker, 18 years experience their attitudes and beliefs, especially in regards to working in teams, problem-solving, accepting constructive criticism, effective daily interactions and the importance of counseling

\section{MONITORING AND SUPERVISION}

The CDPHC program utilized different evaluation mechanisms (including an on-site and off-site monitoring and supervision system that incorporated elements of the wholesite training supervision approach) in order to assess how well the integrated training system functioned in the field and to ensure that trainers and health providers complied with the clinical 
protocols and guidelines provided. This monitoring and supervision system also provided information and feedback on problems health providers faced at their clinics.

On-site supervisors were identified by CDPHC, in collaboration with NGO program managers, who conducted frequent site visits for follow-up support to the providers. This method of supportive supervision ensured that the skills gained from the training were constantly upgraded and that the existing clinic management systems supported the new skills health providers had acquired. The supportive supervision visits focused on constructive feedback, coaching, encouragement, understanding of constraints,

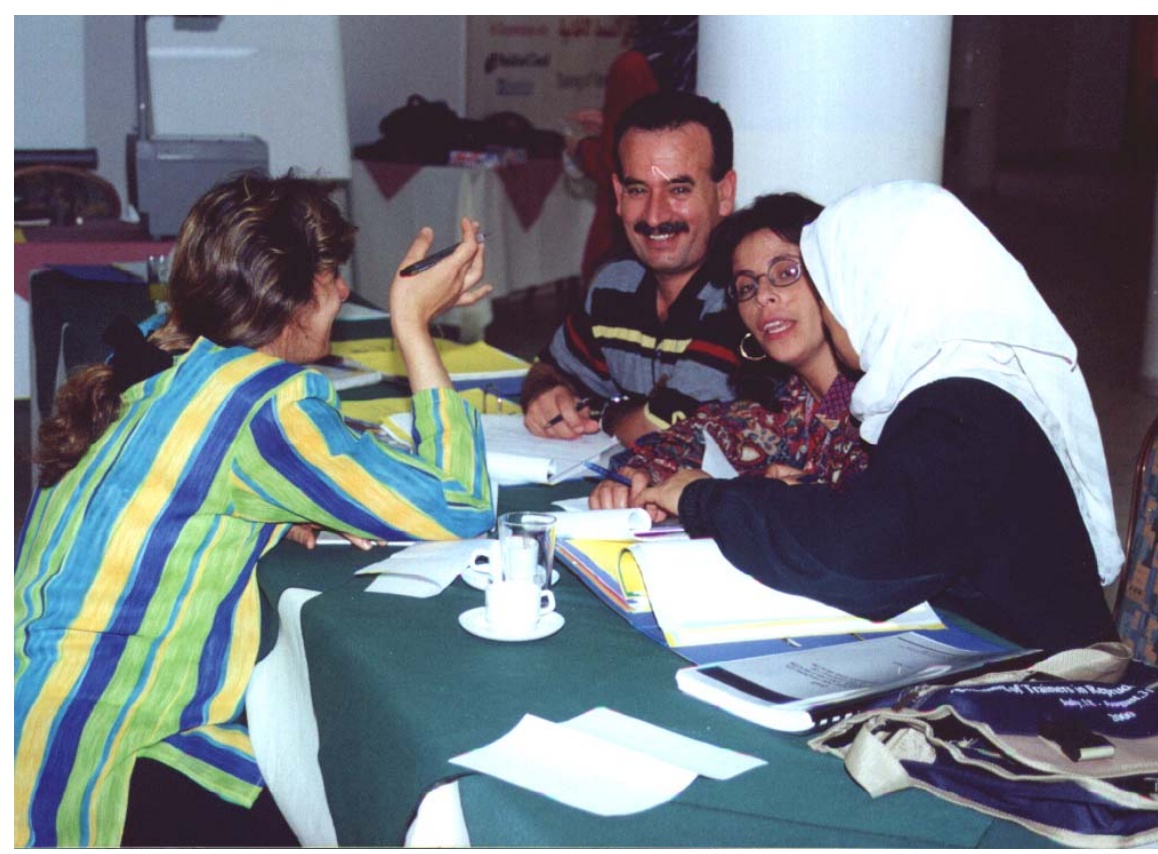
and problem solving. These visits were also an important element in individual and collective performance improvement. On-site supervisors used the same competency-based performance checklists that were developed during the training workshops to evaluate trainees' knowledge, skills, and practices.

In addition to on-site supervisors, each clinic was supposed to be visited at least three times during the life of the project by CDPHC core staff (off-site supervisors). These field visits, however, were curtailed due to the travel restrictions and closures in the West Bank and Gaza. As part of a contingency plan, CDPHC hired off-site supervisors in the three local catchment areas to monitor the trainees. Weekly telephone calls were arranged between CDPHC and the off-site supervisors to discuss progress and follow-up at PHP clinic sites.

The findings from both on-site and off-site supervisors provided direct feedback to trainees about their performance, allowed for the opportunity to discuss any problems or 
constraints to service delivery (e.g., lack of support staff or supplies), and provided CDPHC and supervisors with key information on the adequacy of the training and its appropriateness for local

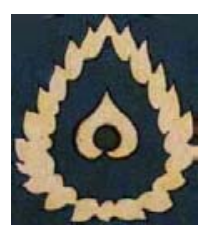
conditions. Most importantly, it also determined the need for refresher training.

\section{POST-TRAINING EVALUATION}

CDPHC conducted a post-training evaluation to measure the impact of the training program in June and July 2001. The results from this evaluation assisted in determining the need for refresher training and identified continuing gaps and weaknesses in the knowledge and skills of health providers previously trained under the PHP. All available health care providers who participated in the training program in both the West Bank and Gaza were included in the evaluation, except for those who were unavailable (e.g., traveling abroad, had resigned from their jobs, or were on leave at the time of data collection).

The post-training evaluation included in-depth interviews with staff and observations of clinical practices. Qualitative interviews were also conducted at clients' homes to assess the quality of the postpartum care and counseling received during postpartum home visits. A total of 40 health care providers were interviewed, 29 in the West Bank (three physicians, four nurses and 22 community health workers) and 11 in Gaza (one physician, five nurses, and five community health workers). The in-depth interviews gathered information on the following types of questions:

을 Did the training improve the health care providers' knowledge and skills?

을 What are the work-related barriers and obstacles that prevent health care providers from offering good counseling?

을 Were the training materials helpful from the providers' point of view?

을 What recommendations do health providers have for additional training that would improve the quality of health care services?

An observation checklist was used to evaluate the skills and performances of health care providers in the following areas: pelvic examinations; antenatal care and counseling; postpartum care and counseling; and family planning services and counseling, including IUD insertions. A total of 34 observations were conducted in the West Bank (twelve antenatal counseling sessions, 
two pelvic examinations, two IUD insertions, twelve family planning counseling sessions, and six postpartum home visits) at both PFS and UPMRC clinics.

To gain more insight and understanding from the in-depth interviews and the clinic observations, focus group discussions were conducted in both West Bank and Gaza in November 2001. Focus group participants were recruited randomly from the list of health care providers who had been previously interviewed. A total of three focus group discussions were conducted, one in the West Bank for community health workers and two in Gaza (one for physicians and nurses and the other for community health workers). Semi-structured interviews were also conducted with two physicians from the West Bank.

\section{A. In-depth Interviews and Observation Findings in the West Bank}

All of the providers reported that the training improved their professional knowledge as well as their counseling and interpersonal skills and they noticed an improvement in their work after the training. Almost all of them found the training materials helpful and reported that they were complying with the clinical protocols and guidelines provided during the training. Only two respondents (senior community health workers) reported that the training did not improve their professional skills.

Most respondents (23 out of 29) reported that the training improved their knowledge about postpartum and antenatal counseling and about half (14 out of 29) said that the training improved their knowledge about family planning. About one-third of the participants (specifically, community health workers) reported being better able to take blood pressure measurements. One-sixth of them reported having improved their infection prevention, communication and counseling, and postpartum home visit skills.

\section{Work-related Barriers to Providing Quality Services}

About 60 percent of community health workers reported that transportation was a key barrier to providing quality services. Many community health workers reported difficulties in conducting home visits because the village areas they covered were too large. Other work-related barriers include: inadequate equipment; shortage of nursing staff, lack of designated private areas/spaces for counseling sessions, large caseloads and inadequate space for providing health 
services; unavailability of important drugs; and lack of electricity and/or telephone services in some clinics. In general, a number of staff members indicated that physical conditions were not always conducive to the provision of quality service for clients.

One-half of the community health workers reported that time constraints prevented them from providing adequate counseling. Six of the community health workers reported the lack of a private area/space as a barrier. Three community health workers reported that clients refused counseling and two said that other staff provided such services. Only two of the providers said that they were not comfortable with their skills.

\section{Training Materials}

Respondents reported that the most helpful materials were related to antenatal and postpartum care, family planning, breastfeeding, counseling and home visits.

\section{Additional Training Needs}

The clinical training provided under the PHP focused on essential information and basic clinical skills related to the provision of safe, high-quality antenatal, postpartum, family planning and selected reproductive health services. The training included the following information: improved and upgraded protocols and standards for antenatal, postpartum, and family planning services; integrated breast and cervical cancer early detection services and referral procedures at the clinic level; infection control standards and practices; counseling and communication skills; and quality management and clinical supervision principles.

Although providers reported that the training improved their knowledge and interpersonal skills, about half of the trainees (mostly community health workers) reported the need for additional training in maternal and child psychosocial health, emergency delivery, and postpartum depression. Physicians reported needing additional training in providing ultrasound tests and nurses asked for additional training in IUD insertion. 


\section{Observations of Antenatal Care Services}

Trained data collectors observed twelve antenatal counseling sessions conducted at nine PHP clinics in the West Bank (seven at PFS and five at UPMRC). The sessions ranged from 10 to 25 minutes depending on the health care provider's workload and profession. Most of the providers that were observed had between seven and 11 years of working experience. Only one provider had one year of experience.

In all of the consultations observed the providers treated their clients with respect. More than half of the health care providers assured clients of confidentiality. Only one provider did not see his client in private. However, most of the providers did not use the PHP family planning flip chart or other visual aids while counseling their clients and only three out of 12 gave their clients information, education, and communication (IEC) materials to take home.

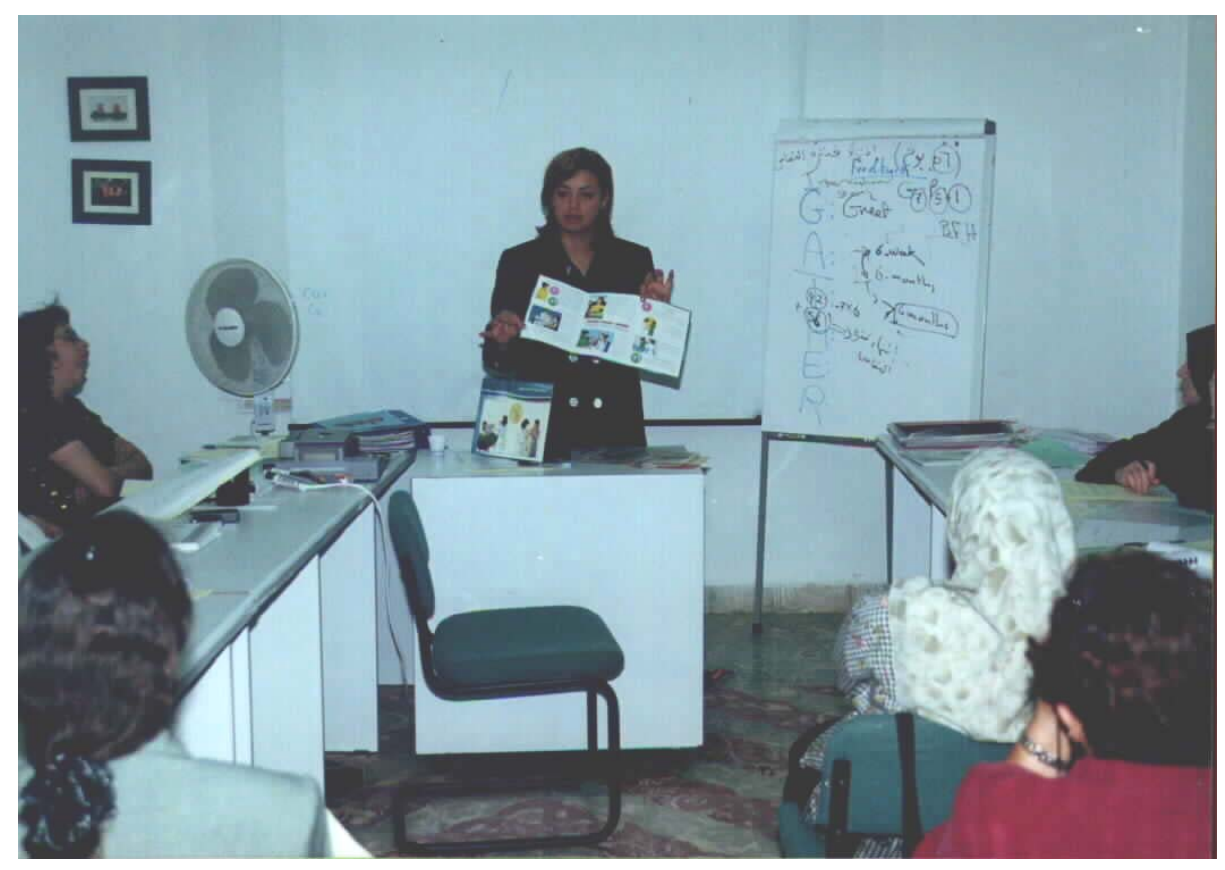

The majority of health care providers counseled their clients about exclusive breastfeeding and about the types of food to eat during pregnancy. In slightly over half of the consultations observed, clients were also told about family planning as well as about warning signs during pregnancy, while only one out of the twelve clients were observed to have told about the dangers of premature rupture of the membrane. About half of the providers counseled their clients about hygiene and exercise, administered or referred their clients for tetanus toxoid, and two-thirds prescribed or provided iron/folic acid tablets.

The results shown in Table 1 indicated that all of the health care providers measured their clients' blood pressure and weight during routine physical examinations. However, only 
one-half looked for signs of edema (especially around the ankles). Approximately one-third of the providers washed their hands with soap before performing a

pelvic exam. Reports from the focus groups

discussions in both the West Bank and

Gaza indicated that physicians did not

routinely wash their hands before a pelvic

examination because some clinics did not

have sinks inside the examination rooms.

In these cases, physicians relied on sterile

gloves for each client.

Three out of the 12 consultancies

that were observed included a breast exam.

The in-depth interviews and focus groups

discussions also revealed why many
Table 1: Routine Clinical Examination for Pregnant Women (taken from clinic observations)

\begin{tabular}{|l|l|l|l|}
\hline Did the provider: & Yes & No & Total \\
\hline Measure the client's height & 5 & 7 & 12 \\
\hline Weigh the client & 11 & 1 & 12 \\
\hline $\begin{array}{l}\text { Apply systemic examination (heart, } \\
\text { GI, etc.) }\end{array}$ & 5 & 7 & 12 \\
\hline Take the client's blood pressure & 12 & 0 & 12 \\
\hline $\begin{array}{l}\text { Wash hands with soap before pelvic } \\
\text { exam }\end{array}$ & 4 & 8 & 12 \\
\hline $\begin{array}{l}\text { Examine eyes and the palm of a hand } \\
\text { for pallor }\end{array}$ & 5 & 7 & 12 \\
\hline $\begin{array}{l}\text { Apply pressure to look for edema } \\
\text { especially around the ankle }\end{array}$ & 6 & 6 & 12 \\
\hline Examine the vulva to check for STIs & 1 & 11 & 12 \\
\hline Use clean gloves (if vaginal exam) & 3 & 9 & 12 \\
\hline Perform or refer for urinalysis & 7 & 5 & 12 \\
\hline Perform or refer for haemoglobin & 8 & 4 & 12 \\
\hline $\begin{array}{l}\text { Palpate abdomen for size of } \\
\text { pregnancy and lie }\end{array}$ & 6 & 6 & 12 \\
\hline Perform breast exam & 3 & 9 & 12 \\
\hline Listen to fetal heartbeat & 7 & 5 & 12 \\
\hline
\end{tabular}

physicians did not perform breast exams. Most physicians reported that clients with breast

problems were identified when they first registered at the clinics and were carefully followed during each of their consecutive visit. Clients without breast problems are not routinely examined during each visit.

\section{Observation of Pelvic Examinations}

Two pelvic examinations conducted by

female physicians were observed during the data collection period. The examinations took approximately five minutes to complete. Although both physicians did ensure the client's privacy and
“ Because of our workloads we sometimes forget or can't comply with all protocols. For example we do not always ask clients to take deep breaths and rest while performing the actual procedure."

Physician, 10 years of experience 
used sterilized instruments and gloves for each exam, the providers did not wash their hands with soap before the pelvic exam, nor did they tell their client to take slow, deep breaths and to relax all muscles during the actual procedure. When asked about these observations later (during the in-depth interviews) the same providers reported that sometimes, because of workload and inadequate physical conditions--including both political and economic reasons--they just could not comply with proper examination procedures.

\section{Observations of IUD Insertions and Family Planning Consultations}

Only two physicians were observed inserting an IUD. Both followed infection control protocols and guidelines during the procedures. They ensured their clients' privacy and reconfirmed new clients' method choice. They washed their hands with soap and water before each exam and used sterilized instruments and gloves. They also asked clients to take slow, deep breaths and relax all muscles before using the speculum and/or conducting the bimanual examination. However, they did not interact or communicate fully with their clients. For example, they neglected to counsel clients about the need to rest for at least 15 minutes after the insertion and to review the signs for IUD expulsion.

Twelve observations (one nurse and 11 community health workers) of family planning services were conducted in 11 PHP clinics. All of these providers greeted their clients, treated them with respect and asked about their previous use of family planning methods. Clients were counseled on their preferred or most-suitable method. They were also told about the effectiveness of the method, its advantages (including protection from STIs and HIV/AIDS), disadvantages, and side effects.

\section{Observations of Postpartum Home Visits}

Six community health workers were observed while performing postpartum home visits at six different PHP clinics in Jenin. All community health workers greeted their clients, obtained a postpartum history and reviewed information about the home environment, serious health conditions, breastfeeding, discomfort or pain, and needs for contraception. The community health workers measured their clients' blood pressure and

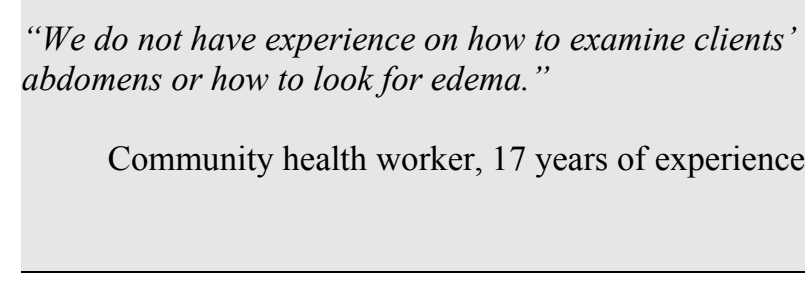


one-half of them measured their clients' weight. None of the Community Health Workers performed abdominal or uterine examinations and only six examined their clients' extremities. None of them requested a vaginal discharge specimen.

\section{B. In-depth Interviews And Observation Findings In Gaza}

A total of 11 health care providers were interviewed using unstructured, in-depth questioning techniques in Gaza (five community health workers, one physician, and five nurses). Similar to the findings from the West Bank, all health care providers in Gaza reported that the training improved their professional knowledge and skills, particularly in the areas of counseling and interpersonal skills. They all also noticed improvements in services after the training and found the training materials helpful in their work at the clinics. They all reported that they were complying with clinical protocols and guidelines provided during their training. In addition, all of them said that they needed additional training.

The key areas in which Gaza health care providers perceived that the training improved their professional knowledge were in family planning, sterilization, communication skills, and antenatal counseling. When asked about improvements in their professional skills, most of the health care providers thought that the training improved their communication, infection control, and management skills. All of the community health workers in Gaza reported difficulties in conducting postpartum home visits. This was mainly

"If the client has any problem, she will tell us about it without the need to perform the exam. Sometimes it is difficult to examine her because there are too many people around her especially after delivery."

Community health worker, 18 months of experience

because the addresses of clients were not recorded accurately and transportation was

problematic. Some of the community health workers also reported that their roles and positions were not accepted in Gaza.

\section{Observations of Antenatal Care Services}

Three doctors, one nurse, and one community health worker were observed by data collectors while performing antenatal counseling sessions for clients at three clinics in Gaza. Data collectors noted that all the health care providers assured clients of confidentiality, treated clients with respect, and asked clients about the number of antenatal visits they had made to the 
clinic. Most of the providers (four out of five) also encouraged clients to ask questions and counseled their clients in private. However, similar to their colleagues in the West Bank, none of the Gaza providers that were observed used family planning flip charts or other visual aids during the sessions. In addition, none of the providers gave their clients any of the IEC reading materials produced by the project.

These health care providers initiated most of the discussions during the antenatal counseling session. Most providers asked their clients about their obstetric histories and about pregnancy complications (if any). They also asked their clients about hypertension and diabetes. Unlike their counterparts in the West Bank, most of the providers observed in Gaza did not counsel their clients about exclusive breastfeeding and few discussed the types of food to eat during pregnancy. More than half of them prescribed iron/folic acid pills to their clients; however, none of them mentioned the side effects of taking these pills. Although more than half of them told their clients about danger or warning signs during pregnancy, yet only one out of five discussed the dangers of premature rupture of the membrane, fever, reduced or accelerated fetal movements. None of the providers administered or referred their clients for tetanus toxoid or counseled their clients about STI and HIV/AIDS prevention. It is interesting to note that only two of the five providers suggested delivery at a health facility to their clients.

When observing routine physical examinations of pregnant clients, data collectors noted that approximately half of health care providers weighed the client, performed or referred them for urine analysis, haemoglobin tests, palpitated their clients' abdomens for size and lie of pregnancy, and listened to the foetal heartbeat. Only one provider measured the client's blood pressure. None of them performed breast exams or checked for edema.

\section{Observation of Pelvic Examinations}

Data collectors in Gaza observed four pelvic examinations, each of which lasted an average of five minutes and was carried out by female physicians only. Data collectors observed that all of the physicians used sterilized or disinfected instruments and new gloves for each examination, and prepared all instruments before the actual exam. All physicians ensured their

client's privacy and followed accurate clinical procedures (inspected the external vagina, the cervix, the vulva, vaginal mucosa, and performed bimanual exams gently and without 
discomfort) for the pelvic exam. However, only one of the four physicians washed her hands with soap before the actual procedure.

\section{Observations of Postpartum Home Visits}

Two postpartum home visits were observed at clients' homes in Gaza. One nurse and two community health workers conducted the home visits. Both the nurse and the community health workers greeted clients, explained the purpose of the visit and obtained the obstetric and medical history of the client. They also obtained the date of delivery of the baby. They asked about the mother's emotional status, sleeping patterns and rest, the home environment, activity level and exercise, appetite and fluid intake, bladder and bowel function, breastfeeding, lochia, and the need for contraception. None of the providers asked mothers about seizures or convulsions or about sexual activity due to cultural sensitivities.

Because of limited resources at the UHWC clinics, the nurse and community health workers did not carry any instruments with them while conducting the home visits. Both the nurse and the community health workers examined clients' breasts and abdomen. However, only the nurse measured clients' blood pressure, examined the uterus and extremities, and performed or referred client for haemoglobin tests.

\section{Results of Focus Group Discussions in the West Bank}

A focus group

discussion for 10

community health workers

and one nurse, and two

semi-structured interviews

with physicians were

conducted in November

2001 to gain more insight

into the results of the in-

depth interviews and

observations as reported

above.

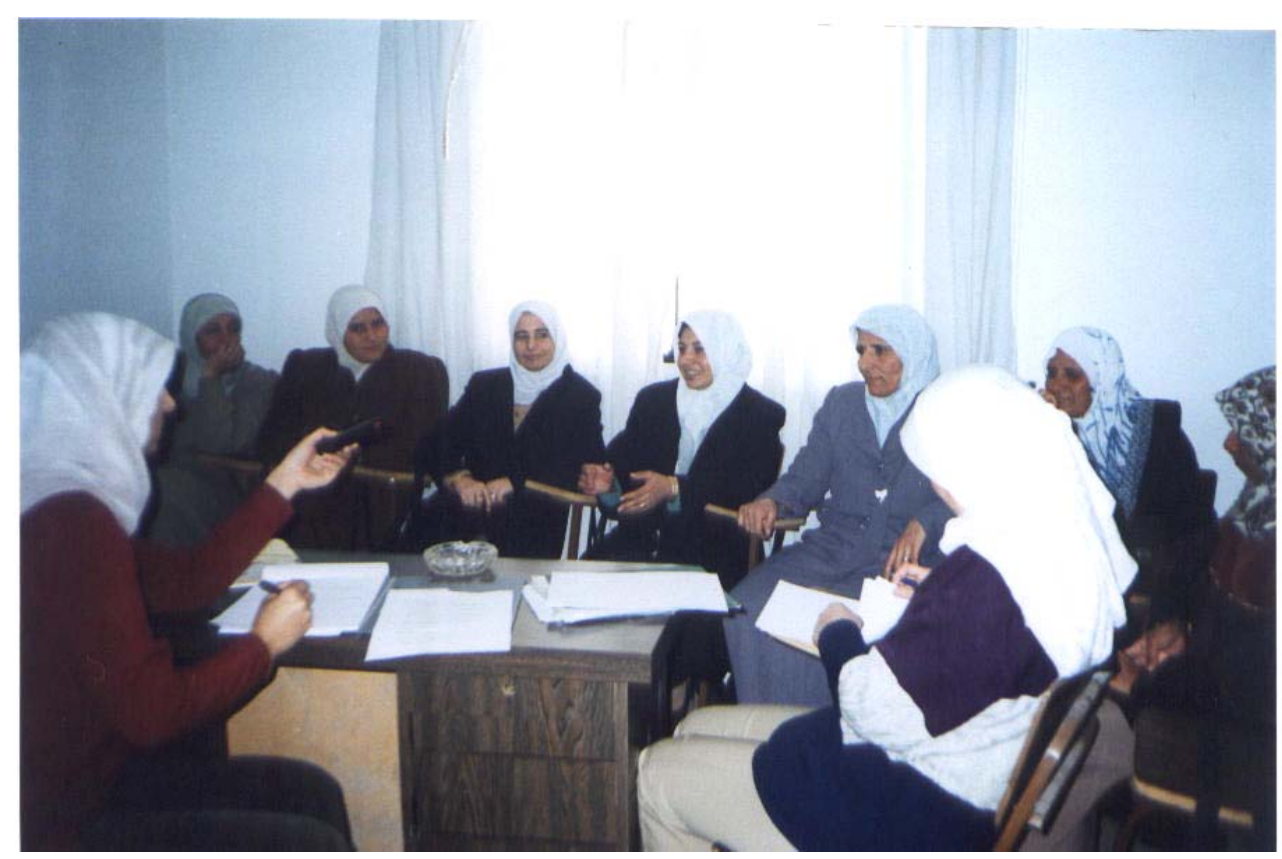


The results from the second round of qualitative data collection reveal that health care providers believe that the training received from $\mathrm{CDPHC}$ improved their overall knowledge and skills (particularly in the areas of antenatal and postpartum care), and upgraded the information they had about reproductive health services.
"The training taught us how to communicate with clients--we gained knowledge about the advantages and disadvantages of each family planning method and which method is most suitable for each client."

Community health worker, 17 years of experience

"The training increased our knowledge and skills and made us pay attention to and focus on communication and counselling services for clients. Client caseloads have since increased, especially for postnatal care and family planning. This indicates that the quality of health care services at my clinic has improved and become more comprehensive and holistic."

Physician, 10 years of experience

Some providers reported that the CDPHC training helped them organize their work and improved their self-esteem and self-confidence, particularly when providing information and education to clients.

Data from the focus group discussion and the semi-structured interviews also indicate that all health care providers believe that the political instability, travel restrictions, and closures

"Many community health workers work outside of their own villages and need to pass through many side roads, detours or checkpoints in order to get to their clinics. Transportation is one of the most difficult problems that community health workers face."

Community health worker, 17 years of experience between cities and villages have severely affected the delivery of services and the quality of care provided in the PHP clinics. The ongoing situation has led to increased workloads during abbreviated hours and new responsibilities and duties, such as first aid and emergency care services. Transportation

and mobility have become extremely difficult and some NGOs have had to create mobile health clinics to serve remote areas or villages that do not have access to health clinics because of the closures and checkpoints.

Health workers also reported that they could not offer comprehensive counseling to clients because of increased workloads, shortages in staff, and the unavailability of private space to conduct counseling sessions. Therefore they focus on providing clients
"The workloads at UPMRC clinics especially Meithaloun Clinic are heavy and staff do not get enough time or sometimes forget to counsel the client about very important topics. In addition, there is no private space to counsel the client. Sometimes men wait with their wives at the clinics so it is not possible to counsel clients in the waiting room areas especially on sensitive topics."

Physician, 18 months of experience 
with essential information. Counseling clients about Pap smears and breast self-examinations were not considered priorities. Providers believe they still need to improve communication and counseling services at the clinics.

Several providers in the focus group discussion also reported that unmarried and young community health workers were shy about counseling clients on sensitive topics (e.g., sexual life after delivery), therefore, they are not providing adequate counseling, especially during postpartum home visits. Traditional and cultural norms dictate that it is unacceptable for

"When the client knows that the community health worker is unmarried she wonders how she knows about such topics without practical experience and this is culturally not acceptable. I remember when I was young if my mother wanted to discuss such topics with other women she did not allow me to sit with them because I was young and unmarried. I think this principal also applies to unmarried community health workers, if they want to discuss such issues, the client may think that this is not her responsibility or duty."

Nurse, 23 years of experience unmarried women to discuss such topics, particularly with married women.

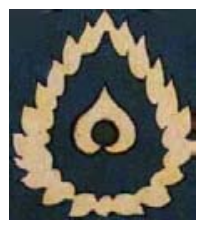

\section{REFRESHER TRAINING}

All providers who demonstrated a need for additional training were provided with refresher training. Several workshops were organized and conducted by CDPHC in the

West Bank and Gaza during the lifetime of the training program. Each workshop was three to four days long and accommodated all categories of health providers.

The curricula for the refresher training courses were tailored to the feedback/data collected from monitoring and supervision

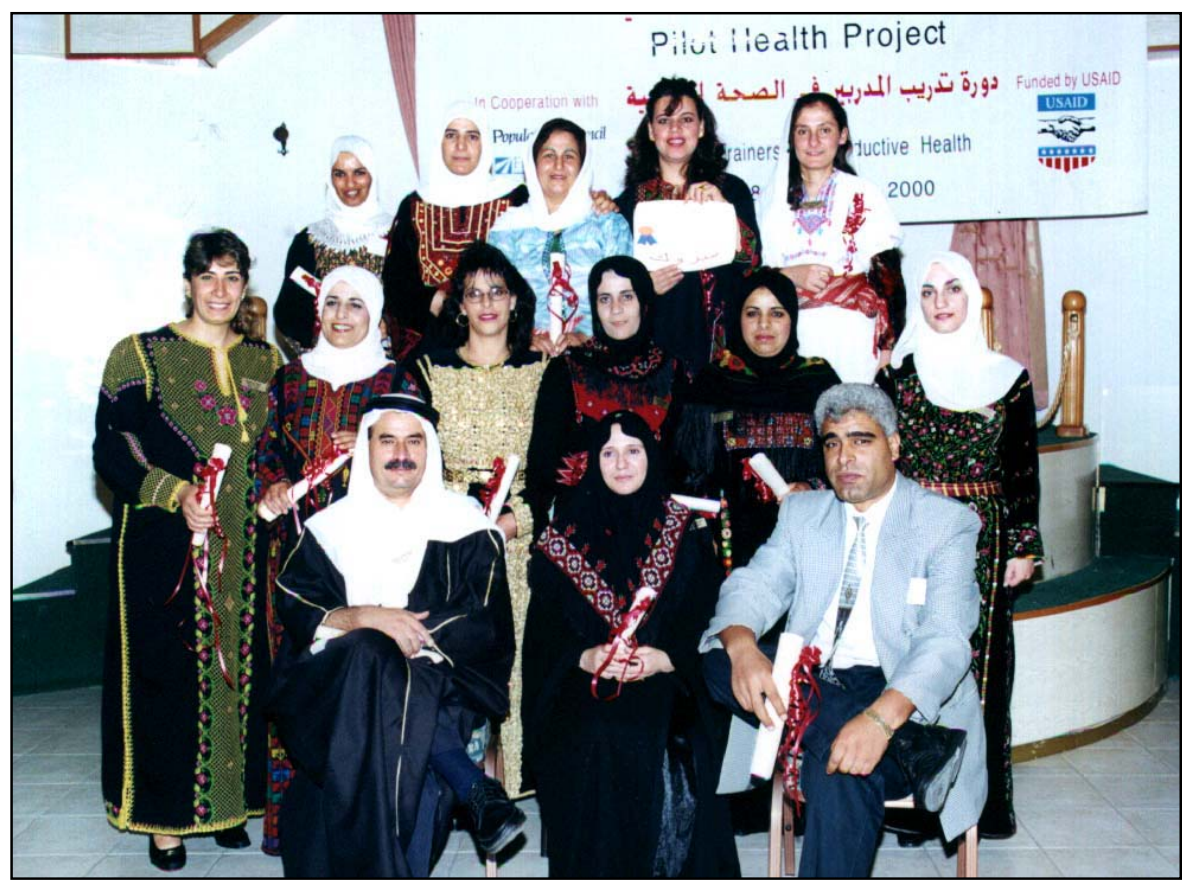


field visits (i.e., the observed and measured performance of health providers after they had received training). The workshops covered a number of topics. The most important topics covered were the following:

\section{Refresher training for community health workers}

- West Bank: Psychosocial and economic problems facing women during antenatal and postnatal periods, and sexual life during pregnancy and after delivery.

- Gaza: Prenatal and postnatal care; family planning; counseling and communication skills.

\section{Refresher training for physicians and nurses}

- Gaza: Nutrition for newborns; communication skills; early detection of cancer, puerperal sepsis, and postpartum depression.

The findings generated from the post-training evaluation conducted in early November 2001 revealed the need for additional training sessions for health providers in both the West Bank and Gaza. These additional training workshops were conducted in November and December 2001 and covered the following topics:

\section{Additional refresher training sessions for community health workers}

- West Bank: Communication skills; counseling on hygiene, exercise, Pap smears and breast self-exams; mother and child psychosocial health; management skills; and immunizations.

- Gaza: Emergency deliveries; measuring blood pressure and body temperature; communication and counseling skills; mother and child psychosocial health; management skills; immunizations; and infection control.

\section{Additional refresher training for physicians}

- Gaza: Counseling and communication; neonatal care; performing ultrasounds; infection control; Pap smear; and breast self-examination. 


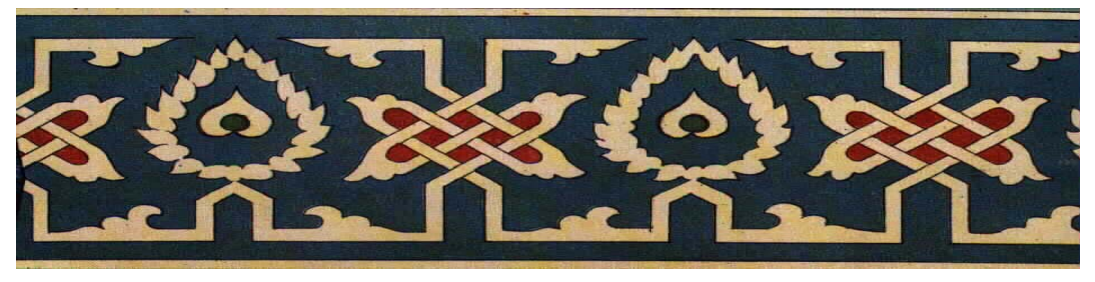

\section{CONCLUSIONS AND RECOMMENDATIONS}

The training provided by CDPHC for the PHP has positively influenced the knowledge and skills of health providers as well as the quality of services at the PHP clinics. Nevertheless, there is an ongoing need to strengthen existing health care services at the primary level. Additional training courses should be planned and conducted to further reduce the gaps and weaknesses identified by the post-training evaluation. Furthermore, clients and medical staff need a suitable physical environment for the provision of quality services, which requires a better level of preparedness of health facilities.

\section{Lessons Learned}

1. Developing physical facility checklists would help optimize and standardize the quality of service by increasing the preparedness of health facilities.

2. Defining roles and responsibilities for various categories of service providers is essential, especially for community health workers.

3. Doctors' sense of safety is a priority so they can move between health facilities.

4. Community health workers should be well informed about the value of antenatal and postpartum counseling, specifically regarding breastfeeding, weaning and family planning.

5. Consensus-building and a participatory approach among PHP partners at all levels is key to successful implementation of program activities.

6. Adopting highly participatory and inclusive management strategies is essential for training programs that involve multiple service delivery organizations since training programs have to meet everyone's expectations and particular needs.

7. The successful implementation of a training program with multiple partner agencies requires strong commitment and sufficient institutional, financial and administrative support of all agencies.

8. Implementing agencies need to be flexible given the unstable environment and travel restrictions. Contingency plans involving program and/or budget changes need to be developed to work around barriers and accomplish activities as best as possible. 
9. Training is a continuous process that requires updating and monitoring. Building in strong supervision systems and refresher training activities is essential for ensuring sustainability at the clinic level.

10. Monthly meetings (via a coordinating council or similar body) are an important method to share information, provide progress updates, build consensus and support, and attain active participation.

11. The formulation of sub-committees/technical review committees to work on specific project activities and/or products is essential.

\section{Recommendations}

1. Continuous training on existing and/or new topics is recommended in order to sustain and improve the knowledge and skills of health care providers.

2. The MOH, NGOs and UNRWA need to coordinate more closely to avoid duplication of services in some areas of the West Bank and Gaza and to better complement each other's work.

3. Additional BCC materials (brochures, posters and audio-visual aids) need to be distributed to PHP clinics to complement the counseling sessions conducted by clinic staff and to improve the quality of health education and counseling services. Refresher training on the use of $\mathrm{BCC}$ materials should be routinely provided to staff to highlight the importance of including these materials in counseling sessions.

4. More health care providers need to be recruited to accommodate the high caseloads and increased workloads at the clinics.

5. Additional renovations and equipment need to be provided to those clinics that still need further upgrading (e.g., private space for counseling, sinks and adequate water supplies).

6. Adequate supplies of contraceptives need to be procured for all clinics to ensure broad method mix.

7. Essential supplements such as iron, vitamins and calcium pills should be provided free of charge to women during pregnancy. 
8. Supportive supervision mechanisms (on-site supervisors, monitoring and supervision visits) need to be established and maintained. Supportive supervision visits should focus on constructive feedback, coaching, encouragement, understanding of constraints, and help with problem solving. 


\section{REFERENCES}

Center for Development in Primary Health Care. Al Quds University. 2000. A Manual for Training in Maternal and Child Health in the Context of Reproductive Health. Palestine.

Ismail, N. and M. Shahin. 1996. Family Planning and Women's Reproductive Health Survey in the West Bank. Planning and Research Center: Ramallah, Palestine.

Palestinian Central Bureau of Statistics, 1997. The Demographic Survey in the West Bank and Gaza Strip: Final Report. Ramallah, Palestine.

Palestinian Ministry of Health, 1997. Annual Report 1997. Gaza/Nablus, Palestine.

UNFPA, 2000. The Occupied Palestinian Territory: Country Population Assessment. Ramallah, Palestine.

Younis, N. and N. Hassanien. 1999. Consultancy Report: Assessment of Standards of Health Care Services: Pilot Health Project. Cairo.

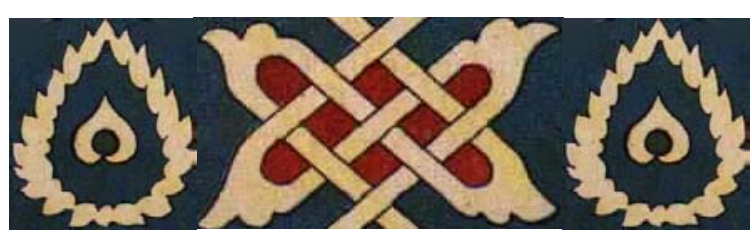

